\title{
LOS ESTUDIOS DE RIESGOS NATURALES Y ANTRÓPICOS A TRAVÉS DE CUATRO DÉCADAS EN LA REVISTA GEOGRÁFICA DE AMÉRICA CENTRAL
} (1974-2015)

\author{
NATURAL AND ANTHROPIC RISK STUDIES IN FOUR \\ DECADES IN THE GEOGRAPHICAL JOURNAL OF \\ CENTRAL AMERICA (1974 - 2015)
}

\author{
Adolfo Quesada-Román ${ }^{1}$ \\ Universidad Nacional de Costa Rica, Costa Rica
}

\begin{abstract}
RESUMEN
El objetivo de este trabajo es identificar la influencia tanto internacional como nacional de las tendencias epistemológicas y técnicas en la gestión del riesgo de desastres (GRD) en Costa Rica, para lograrlo, se analizaron las investigaciones publicadas como artículo en la Revista Geográfica de América Central entre 1974 y 2015, de las cuales fueron identificados 114 documentos con tópicos asociados a los riesgos naturales y antrópicos. Se lograron catalogar ocho clases temáticas: amenazas sísmicas, amenazas volcánicas, amenazas por procesos de ladera, amenazas hidrometeorológicas, cambio climático, degradación ambiental y erosión de los suelos, vulnerabilidad, y epistemología de la gestión del riesgo de desastres en Costa Rica y Latinoamérica. Como resultado de esta investigación fue posible identificar un aumento considerable de trabajos asociados a la GRD a partir de finales de la década de 1980 con una clara diversificación en las temáticas abordadas.
\end{abstract}

Palabras clave: gestión del riesgo de desastres, amenazas naturales, vulnerabilidad, riesgos naturales, riesgos antrópicos

1 Escuela de Ciencias Geográficas, Universidad Nacional de Costa Rica, Costa Rica. Correo electrónico: adolfo.quesada.roman@una.cr; adolfo.quesada@gmail.com

Fecha de recepción: 1 de agosto de 2016

Fecha de aceptación: 22 de agosto de 2016 


\begin{abstract}
The aim of this work is to identify the influence of the international and national epistemological trends and the techniques in the risk management of disasters (RMD) in Costa Rica. To do this, the journal papers of the Geographical Journal of Central America between 1974 and 2015 were analyzed -114 included topics related to natural and anthropogenic risks. They were classified into eight thematic classes: seismic hazards, volcanic hazards, slope processes hazards, hydrometeorological hazards, climate change, environmental degradation and soil erosion, vulnerability, and epistemology of risk management disasters in Costa Rica and Latin America. As a result of this work, it was possible to identify a significant increase in jobs related to the GRD from the late 1980 's, with a clear diversification of the issues addressed.
\end{abstract}

Keywords: Disaster risk management, natural hazards, vulnerability, natural risks, anthropic risks

\title{
Introducción
}

Los distintos territorios que conforman la superficie terrestre son producto de una intrínseca relación entre el ser humano y la naturaleza a partir de respuestas culturales, socioeconómicas, políticas y geográficas. De acuerdo a Toscana (2006), la primera discusión social sobre desastres en Occidente se dio en torno al terremoto de Lisboa en 1755, el cual tuvo una magnitud de 8.5 en la escala de Richter y se presentó el 1 de noviembre de 1755 (día de Todos los Santos) cuando había muchas personas en misas, además, se produjo un tsunami de casi $15 \mathrm{~m}$ de altura; posteriormente, la ciudad ardió en fuego durante 6 días, para culminar con intensos saqueos por parte de algunos sobrevivientes, el desastre tuvo un total de 15 mil muertos.

Dicha disertación se dio no sólo por la magnitud e intensidad del sismo sino por el contexto cultural y las acciones para reparar los daños; sino que este evento llamó la atención de notables filósofos de la época entre ellos Voltaire, Liebniz, Pope, Kant y Rousseau, de donde se origina la primera discusión científica social de los desastres. Este evento tuvo importantes implicaciones a largo plazo, entre ellas el significado de una catástrofe dentro del contexto de la Ilustración, ya que fue un tema de debate intelectual. Rousseau argumentó que la localización de la ciudad la hicieron susceptible a sufrir sismos y tsunamis; y el patrón urbano y el tipo de construcción la hicieron susceptible a daños por sismos, lo que se podría considerar como las bases de la vulnerabilidad. Además, es de recalcar que fue la primera vez que un Estado aceptó la responsabilidad de asumir las consecuencias del sismo, dado que Portugal movilizó la respuesta ante la emergencia y desarrolló un esfuerzo colectivo para la reconstrucción 
(Toscana, 2006). Este evento fue un hito histórico para forjar conceptos y teorías basados en la ciencia y un incipiente desapego de las creencias religiosas o esotéricas que acusaban a castigos divinos o sobrenaturales sus desgracias asociadas a los desastres producto de la dinámica terrestre.

Las formas actuales de tratar los desastres para su estudio tienen sus bases conceptuales en los trabajos pioneros que definieron dos líneas de investigación desde mediados del siglo XX: la geográfica y la sociológica. La línea geográfica se desarrolla dentro de la Escuela de Ecología Humana de la Universidad de Chicago a finales de la década de 1920 por Dewey, quien planteó que la humanidad existe en un mundo natural innatamente peligroso lo que es inseguro para los humanos, quienes viven en un círculo vicioso de problemas ambientales sin resolver (Toscana, 2006). Estos postulados fueron la base para la obra de Gilbert White a mediados del siglo XX, entre 1930 y 1960, basados en análisis de zonas de inundación y otras amenazas naturales en Estados Unidos, cuando inicia con las distinciones entre "fenómeno natural peligroso", "desastre", y "riesgo" (White, 1942, 1974). Posterior a la Segunda Guerra Mundial y la subsecuente carrera nuclear armamentística se creó un interés mayor del actuar respecto a posibles desastres desde la investigación (Bankoff, 2004).

La línea sociológica se desarrolla a partir de la tesis doctoral de Prince "Catastrophe and Social Change" (1920), que trata sobre la explosión de Halifax, Canadá en 1917; donde se explora el comportamiento colectivo de la población durante la emergencia. No obstante, es durante la década de 1960 que se inicia una corriente sociológica enfocada a la investigación social de los desastres, donde destacaron investigadores estadounidenses como Henry Quarantelli y Russell Dynes, cuyas contribuciones se asocian al comportamiento colectivo y el análisis organizacional en trabajos sobre desastres (Maskrey, 1993). Por su parte, la antropología se ha centrado en el estudio de la experiencia del desastre a partir de la década de 1950; por lo que desde entonces, en el ámbito de la geografía, sociología y antropología se llevan a cabo investigaciones sobre la forma en que los individuos y grupos sociales se adaptan al ambiente mediante el cambio del uso del suelo, reglamentos de construcción y la implementación de diversas tecnologías (Toscana, 2006).

Por muchos años se mantuvo la idea de que los desastres eran eventos extremos inevitables que requerían soluciones puramente tecnocráticas que hacen insuficiente justicia a una genealogía intelectual más compleja; 
no obstante, este paradigma se mantuvo por varias décadas por medio de las Naciones Unidas y agencias de financiamiento multilaterales como el Banco Mundial. Esta condición ha tenido un importante peso en los más altos niveles de toma de decisiones nacionales e internacionales, al punto de declarar la década de 1990 como la Década Internacional de la Reducción de Desastres Naturales (Bankoff, 2004).

De acuerdo a Barrantes y Márquez (2011) a partir de la década de 1970 se ha dado una discusión científica sobre dos distintas formas de abordar el estudio de los desastres: el enfoque tradicional o del comportamiento, originado por la escuela norteamericana de desastrología y el enfoque estructural basado en las experiencias de campo obtenidas por los investigadores en los países subdesarrollados. A estos dos enfoques se le suma un nuevo paradigma nacido en América Latina, impulsado por una visión sistémica, al cual algunos autores le denominan holístico.

Un gran hito en América Latina fue la creación en 1992 de la Red de Estudios Sociales en Prevención de Desastres en América Latina (LA RED), entre sus objetivos se encuentran la promoción de la investigación comparativa sobre la prevención y el manejo de desastres en América Latina, el establecimiento de canales de comunicación entre los investigadores sociales hasta ahora dispersos y la amplia difusión de los resultados de las investigaciones realizadas en la región (Maskrey, 1993).

No obstante, de acuerdo a Lavell (2005) la producción científica de investigadores latinoamericanos en gestión del riesgo ha sido escasa y muchas veces generada solamente en español (o portugués). A esto debe sumarse el "fisicalismo" en América Latina dada la larga trayectoria de las Ciencias de la Tierra y de las ingenierías en la región orientadas hacia el análisis de los procesos físicos o estructurales de mayor relevancia, para el conocimiento de las amenazas y sus impactos en estructuras y edificaciones. En la región, además, han surgido varios paradigmas o marcos analíticos distintos frente al problema del riesgo y desastre, algunos disciplinarios otros multidisciplinarios, que se han quedado en el transitar hacia un camino más de la búsqueda de una meta - perspectiva muy ligada a la problemática del desarrollo y no han resuelto aún la complejidad del fenómeno del riesgo y desastre.

Es importante acotar, que el $95 \%$ de las muertes anuales por desastres ocurridos en el mundo se localizan en Asia y América Latina, especialmente en países en desarrollo o del tercer mundo como Bangladesh, India, China, 
México, Costa Rica, Colombia o Chile, esto se da por las condiciones naturales como su situación geográfica asociada a la Zona de Convergencia Intertropical (altos regímenes lluviosos aunado al paso de ciclones tropicales) y el Cinturón de Fuego del Pacífico (asociado al impacto de la sismicidad y vulcanismo), lo que sumado a las condiciones de vulnerabilidad proporciona las condiciones ideales de riesgo (Alcántara, 2002).

Cabe resaltar que a nivel latinoamericano el estudio de la gestión del riesgo tiene un importante empuje después de 1985 con el terremoto de la Ciudad de México y el lahar del volcán Nevado de Ruiz en Colombia, los cuales dejaron miles de muertos y marcaron un hito para la investigación, inversión pública y concientización de la problemática de los desastres en las economías locales y regionales.

Para el caso de Costa Rica, distintos eventos históricos tuvieron sustancial peso en la conformación de un grupo de expertos y el desarrollo de la temática de gestión del riesgo, entre ellos la temprana conformación de la Comisión Nacional de Emergencias en 1969, y su atención de diversos ciclones tropicales, intensas precipitaciones así como temporales y terremotos con la activación de albergues y respuesta ante dichas emergencias en todo el país durante las décadas de 1970 y 1980 . A su vez, muy importante fue el aprendizaje a partir de dos eventos naturales que tuvieron gran impacto en el país: el huracán Juana en 1988 y el terremoto de Limón en 1991.

La importante labor que por ley le correspondía a la Comisión Nacional de Emergencia, era paralelamente apoyada por la información generada por el Instituto Meteorológico Nacional desde el punto de vista hidrometeorológico. Por otro lado, el estudio de la sismología y vulcanismo del país se deben a la conformación de la Red Sismológica Nacional - RSN (Escuela Centroamericana de Geología de la Universidad de Costa Rica y el Instituto Costarricense de Electricidad) y posteriormente del Observatorio Vulcanológico y Sismológico de Costa Rica - OVSICORI de la Universidad Nacional de Costa Rica, de donde se ha generado información precisa que ha servido de base para el desarrollo de múltiples investigaciones no sólo de la dinámica terrestre sino de su aplicación con la gestión del riesgo y el ordenamiento territorial.

\section{Metodología}

La idea de construir un documento que agrupe los trabajos asociados con amenazas, vulnerabilidad, riesgos y desastres durante la historia de la 
Revista Geográfica de América Central se fundamenta en la necesidad de conocer la evolución de las tendencias conceptuales a lo largo del tiempo de los estudios de estas temáticas. Entender la dinámica terrestre que puede provocar pérdidas económicas y humanas, así como la degradación del medio ambiente; además de estar vinculado de manera directa con la Geografía, se asocia con múltiples ciencias tanto físico-naturales como la geología, hidrología, ingenierías, así como sociales como la antropología, historia, sociología, y las ciencias políticas.

La Revista Geográfica de América Central se publica desde 1974 con la fundación de la Escuela de Ciencias Geográficas de la Universidad Nacional de Costa Rica. Desde sus inicios, esta publicación hemerográfica buscó el desarrollo de trabajos que abarcaran temáticas de las Ciencias de la Tierra, las Ciencias Sociales y las Ciencias Naturales con un enfoque geográfico donde privaran principios fundamentales de la Geografía como la localización, distribución y la inherente relación dialéctica ser humanomedio ambiente. La revista actualmente se despliega en el portal digital de revistas de la Universidad Nacional de Costa Rica y tiene a disposición del público todas sus publicaciones en formato digital desde su nacimiento, puede visualizarse desde la siguiente dirección electrónica: http://www. revistas.una.ac.cr/index.php/geografica.

Se realizó una revisión bibliográfica de todos los artículos publicados en la Revista Geográfica de América Central desde 1974 hasta 2015. Del total de trabajos se eligieron 114 documentos que tenían asociación directa con la temática de riesgos naturales y antrópicos en diversas áreas del conocimiento, los cuales a su vez fueron clasificados en 8 clases: amenazas sísmicas, amenazas volcánicas, amenazas por procesos de ladera, amenazas hidrometeorológicas, cambio climático, degradación ambiental y erosión de los suelos, vulnerabilidad, otros riesgos naturales, y epistemología de la gestión del riesgo de desastres.

Se hizo una revisión de diversas fuentes documentales sobre la historia de la GRD así como las diferentes tendencias en la evolución de los conceptos y teorías a escala global y en América Latina, que lograran tener un vínculo directo con el tipo de productos científicos que se publicaron a través de la Revista Geográfica de América Central a lo largo de cuatro décadas. 


\section{Resultados}

De acuerdo con el estudio de los artículos publicados en la Revista Geográfica de América Central durante cuatro décadas, fue posible desarrollar una clasificación de las temáticas dominantes para los trabajos asociados a riesgos naturales y antrópicos en esta publicación hemerográfica. Se elaboró una sistematización con nueve categorías que engloban los ejes que describen los análisis elaborados a lo largo del tiempo, lo que da un patrón sobre las tendencias epistemológicas y conceptuales que eran abordadas según el contexto histórico, regional, nacional o incluso local asociado a las amenazas, vulnerabilidades, riesgos y desastres.

Las temáticas de los artículos fueron diferenciadas de acuerdo con la cantidad de documentos en cada uno de estos ejes en amenazas hidrometeorológicas, degradación ambiental y erosión de los suelos, amenazas volcánicas, amenazas sísmicas, amenazas por procesos de ladera, epistemología de la gestión del riesgo de desastres, vulnerabilidad, y cambio climático (Tabla 1).

Tabla 1. Eje temático y cantidad de artículos relacionados con riesgos naturales y antrópicos en la Revista Geográfica de América Central (1974-2015)

\begin{tabular}{|l|c|c|}
\hline \multicolumn{1}{|c|}{ Temática de artículos } & Cantidad & Porcentaje \\
\hline Amenazas sísmicas & 13 & 11,40 \\
\hline Amenazas volcánicas & 16 & 14,04 \\
\hline Amenazas por procesos de ladera & 11 & 9,65 \\
\hline Amenazas hidrometeorológicas & 26 & 22,81 \\
\hline Cambio climático & 4 & 3,51 \\
\hline Degradación ambiental y erosión de los suelos & 25 & 21,93 \\
\hline Vulnerabilidad & 8 & 7,02 \\
\hline Epistemología de la gestión del riesgo de desastres & 11 & 9,65 \\
\hline
\end{tabular}

Fuente: Elaboración propia a partir de los artículos consultados (2017).

Entre los estudios de las amenazas sísmicas, resaltan los artículos de Merino (1975) sobre el Terremoto de la Ciudad de Guatemala, Cassertano 
(1979) sobre el cálculo de magnitudes máxima y mínima de sismos, González et al. (1986) sobre un plan para incorporar el riesgo sísmico a los planes de uso del terreno; así como diversos trabajos muy puntuales sobre la sismicidad de diversos sectores del país en temporalidades históricas y durante la década de 1980 con apoyo instrumental durante el I Congreso de Desastres Naturales en 1988, de donde se publicó un número especial en 1992: Parismina (Brenes, 1992), hoja San José 1:200.000 (Güendel et al., 1992), Terremoto de Fraijanes de 1888 y fallas de Alajuela (Morales, Montero y Madrigal, 1992), Potrero Grande y Sierpe (Protti et al., 1992).

Otros estudios asociados con las amenazas sísmicas en Costa Rica son los de Montero et al. (1997) sobre un modelo de simulación de intensidades sísmicas para la microzonificación del país, y Segura et al (2014) con un análisis de la actividad sísmica en 2010 y el resumen de eventos históricos entre 1983 y 2012. Por otro lado, fueron publicados dos artículos de otros países de la región latinoamericana, uno de México (Madrigal et al., 2010), Brasil (Tanizaki \& Azevedo, 2011) y asociados a levantamientos tectónicos de las costas y el impacto de tsunamis (Martínez et al., 2011; Martínez, 2011). Los trabajos asociados a la amenaza sísmica se circunscriben en mayor medida en estudios sobre la sismicidad en áreas específicas con enjambres sísmicos o actividad recurrente siempre acompañados de la instrumentación especializada y la aplicación de modelos para determinar microzonificaciones de la magnitud e intensidades sísmicas; estos estudios se han presentado desde los orígenes de la revista. Además que su producción va en aumento una vez que existe una tecnología mejorada y que los institutos de investigación como el OVSICORI y la RSN (UCR-ICE) pueden mejorar la calidad de sus mediciones y por ende investigaciones.

En el caso de las amenazas volcánicas, desde el nacimiento de la revista esta temática ha tenido una importante relevancia dada la geodinámica volcánica tanto interna como externa en Costa Rica y Latinoamérica. El primer estudio de esta línea de investigación fue presentado por Cevo (1974), el cual analizó las manifestaciones volcánicas en Aysén (Chile) en 1973. Durante los números 5-6 de la revista se publicaron varios trabajos sobre la vulcanología costarricense, entre ellos de las erupciones del volcán Arenal (Bennett \& Raccichini, 1977a; Sáenz, 1977), volcán Poás (Bennett \& Raccichini, 1977b), la actividad del volcán Irazú durante 1723 (Escuela de Ciencias Geográficas-UNA, 1977) y un estudio geológico del 
Cerro Chopo (Mora, 1977). Posteriormente, Bennett \& Wright (1979) y Bennett (1980) estudian los niveles de azufre del cráter del volcán Poás; durante 1980, Borgia \& Cigolini (1980) analizaron la viscosidad y estructura de las coladas del volcán Arenal.

Posteriormente, no es hasta 1992 con los resultados del I Congreso Nacional de Desastres Naturales en 1988 que se retoman estudios volcánicos como las implicaciones de la lluvia ácida en la vegetación del volcán Poás (Alfaro, 1992), síntesis geovulcanológica del volcán Arenal entre 1968-1988 (Alvarado et al., 1992), una histografía del Volcán Poás (Barquero et al., 1992), y sobre los peligros potenciales de la Cordillera Volcánica Central (Soto y Paniagua, 1992). De manera más reciente se estudiaron las percepciones y prevenciones asociadas con el riesgo por lahar en los Diques de Cartago (Barrantes et al., 2008), los callejones de acidificación en tres volcanes activos de Costa Rica (Duarte y Fernández, 2011) y las cuatro erupciones freáticas del Volcán Turrialba entre 2010 y 2013 (Duarte, 2014). Es interesante apuntar que los estudios volcánicos que han sido publicados en la revista están asociados, en su mayoría, con volcanes activos de Costa Rica durante sus fases eruptivas. Los análisis históricos, dataciones radiométricas o la potencial reactivación de sistemas volcánicos no son temas de discusión recurrente en la revista a pesar que la vulcanología es un tema de constante publicación e investigación en el país y la región.

En Costa Rica los procesos de ladera, movimientos en masa o procesos de remoción en masa siempre han sido llamados deslizamientos, sin importar el tipo de movimiento que predomine según su sustrato (rocas, detritos o suelos), velocidad del movimiento, superficie de ruptura o tipo de depósito. Esta problemática ha sido detectada en otros países latinoamericanos dadas las traducciones desde otros idiomas, especialmente del inglés al español (Alcántara-Ayala, 2000). En la Revista Geográfica de América Central se podría decir que los estudios de procesos de ladera nacen en la década de 1990 con el número 25-26 sobre los resultados del I Congreso Nacional de Desastres Naturales en Costa Rica, se publicaron trabajos sobre deslizamientos en Puriscal (Arroyo, 1992a), la inestabilidad de laderas en la zona norte de Alajuela (Mora \& Morales, 1992) y los deslizamientos en la cuenca del río Reventado (Vahrson \& Cartín, 1992).

Estudios de otras regiones del país sobre movimientos en masa fueron hechos en Bahía Culebra, Bahía El Coco (Vahrson \& Arauz, 1993), río 
Banano en Limón (Hernando \& Arauz, 1994), la aplicación de la metodología Mora-Vahrson en la zona más afectada por el Terremoto de Cinchona (Barrantes et al., 2011), y la delimitación de 4600 deslizamientos provocados por el Terremoto de Cinchona (Barrantes et al., 2013). Es importante también indicar que se han publicado trabajos de otros países como Brasil en la comunidad de Divino E. Santo, el barrio de Santa Maria Aracajuse, el río Itoupava y el cerro Campana cerca de la ciudad de Juina la Castaña (Campos et al., 2011; Dos Santos et al., 2011; Volker \& Casagrande, 2011; Zocche \& Peralta, 2011). Los estudios de amenazas por procesos de ladera tienden a tener un crecimiento notable a inicios de la década de 1990, y tienen un resurgimiento muy importante a partir de 2011 hasta la actualidad.

Las investigaciones de las amenazas hidrometeorológicas son las que tienen un mayor número de publicaciones (25) y nacen con la primer divulgación de la revista, donde Govaere (1974) hace un mapa de isoyetas de un evento de precipitación extraordinaria en la cuenca del río Grande de Térraba. No obstante, hasta la realización del I Congreso Nacional de Desastres Naturales efectuado en 1988 y la posterior publicación de dichos trabajos en un número especial de 1992, se analizaron distribuciones de periodos de retorno (Vahrson, 1992), la aplicación del programa CFA88 para eventos meteorológicos extremos (Fallas, 1992a; Fallas, 1992b), riesgos por sequía en el país (Patterson, 1992), riesgo por inundaciones en Costa Rica (Arroyo, 1992b), avalanchas e inundaciones en los ríos de Orosi y Pejibaye (Vahrson et al., 1992a), análisis probabilístico de las lluvias intensas en Costa Rica (Vahrson et al., 1992b), avalanchas e inundaciones por fuertes precipitaciones el río Ciruelas (Vahrson et al., 1992c), el efecto de la variación del coeficiente de escorrentía en la frecuencia de avenidas (Rodríguez, 1992), situaciones climatológicas que causan desastres en Costa Rica (Ramírez, 1992), y una clasificación de fenómenos meteorológicos de desastres en Costa Rica (Zárate, 1992). Otros estudios durante la década de 1990 fueron; a saber, uno sobre el efecto del Fenómeno del Niño en Costa Rica (Chacón, 1993) y otro relacionado con sensores remotos y la zonificación de inundaciones en las cuencas bajas de los ríos Parrita y Limoncito (Arroyo, 1996).

A partir del siglo XXI, la revista tuvo un importante número de artículos sobre amenazas hidrometeorológicas, donde se analizaron desde comportamientos hidrológicos estacionales y su relación con el ENOS en 
la cuenca del río Tárcoles (Quesada, 2001), una zonificación de amenaza por inundación en el valle del río Sixaola (Barrantes \& Vargas, 2011), un análisis de la incidencia espacial de inundaciones y deslizamientos entre 2000 y 2006 (Arroyo, 2011), y el riesgo por inundación en Cahuita (Sandoval \& Chacón, 2011).

Durante 2011, se realizó el XIII Encuentro de Geógrafos de América Latina (EGAL) en Costa Rica, gracias a ello hubo una importante cantidad de estudios asociados a las amenazas hidrometeorológicas en la región, con trabajos que abarcaron temas como la vulnerabilidad espacial climática de la ciudad de Aquidauana en Brasil (Freitas \& Da Anunciação, 2011), la incidencia de inundaciones y deslizamientos entre 1999 y 2009 en Costa Rica (Arroyo et al, 2011), las inundaciones en la ciudad de Belo Monte en Brasil (Cardoso, 2011), las inundaciones asociadas al proceso de urbanización de la ciudad de Juiz de Fora en Brasil (Oliveira y Baptista, 2011), los impactos de la urbanización en el sistema hidrológico y las inundaciones del municipio de Guarujá en Brasil (Pereira y Oliveira, 2011), las alteraciones en los indicadores agroclimáticos en años Niño en el Centro-Occidente de México (Granados et al., 2011), la población afectada por fenómenos climáticos extremos en México (Ortiz y Vidal, 2011), y el estudio de eventos superiores a $100 \mathrm{~mm} / 24 \mathrm{~h}$ en la ciudad de Fortaleza, Brasil (Pereira et al., 2011).

Los estudios de cambio climático han tenido un crecimiento notable en las últimas dos décadas al tener una veracidad científica sobre el aumento de las temperaturas medias de la Tierra en los últimos 250 años a partir de la Revolución Industrial. Los trabajos dedicados a este tema en la revista se circunscriben a solamente cuatro, que se dieron en el EGAL realizado durante 2011 en Costa Rica, con títulos como cambio climático, globalización y nueva división internacional del trabajo (Espinosa, 2011), cambio climático y variabilidad en la dinámica de los ecosistemas de Wirikuta, Municipio de Catorce, México durante 1950 y 2010 (Loyola et al., 2011), geografía física, riesgos socioambientales y cambio climático en Brasil (Silva et al, 2011), y cambio climático, turismo y riesgo de incendios forestales y de pasturas en el sudoeste de la provincia de Buenos Aires en Argentina (Uboldi, et al., 2011).

Los riesgos no solamente son generados por causas naturales producto de la dinámica de la tectónica, vulcanismo, dinámica atmosférica 
o gravitacional, sino que también se asocian a la modificación por parte del ser humano respecto a su medio; es por ello que en este trabajo se integran las amenazas por degradación ambiental y erosión de los suelos. Entre los estudios que resaltan en esta categoría están el trabajo de Cartín \& Ellenberg (1986) sobre las características geomorfológicas, hidrográficas y vegetacionales de ambos lados de la Cordillera Volcánica Central de Costa Rica y se estudian las tendencias catastróficas de no producirse una intervención más enérgica para su protección. Por otro lado, Fassone (1992) explica sobre el desastre visto como una calamidad que agota los suelos y aguas, el estudio de Vahrson (1993) sobre erosión y conservación de suelos en Costa Rica, la erosión de suelos en Cerbatana de Puriscal entre 1990 y 1995 (Hernández et al., 1995), el desarrollo de un modelo geomorfométrico para el estudio de la erosión en cárcavas (Hernández, 1995), la degradación de recursos, pobreza y salud en el cantón de Puriscal (Somarribas \& Patterson, 1997a; Somarribas \& Patterson, 1997b), el modelaje de la erosión de suelos en Costa Rica con el modelo WEPP (Hernández \& Ruiz, 1998), un diagnóstico físico-ambiental para el Plan Regulador del cantón de Esparza (Arroyo, 2008), la evaluación del riesgo ante incendios forestales en la cuenca del río Tempisque (Moraga, 2010), y el impacto de los incendios forestales en la erosión hídrico de la Sierra de Santa Helena en Portugal utilizando la Ecuación Universal de Pérdida del Suelo (Meneses, 2013).

Posteriormente, durante el EGAL de 2011 en Costa Rica se presentaron múltiples trabajos de este eje temático. Entre los estudios presentados están uno sobre la identificación de riesgos ambientales en la bahía de Río Verde en Paraná, Brasil (Cortese et al., 2011), el riesgo por contaminación de los recursos hídricos en Catamarca, Argentina (Segura et al., 2011), lo procesos erosivos asociados con el cultivo de tabaco en Faxinal, Brasil (Cassol \& Floriani, 2011), la historia de obras de contención erosiva en la ciudad de Terra Rica, Brasil (Correia et al., 2011), el proceso de desertificación en el Municipio de Santa Maria de Cambuca, Brasil (Galvão \& Ribeiro, 2011), la degradación del suelo en una zona semiárida tropical en el Valle de Tehuacán-Cuicatlán, México (Hernández et al., 2011), los impactos de erosiones aceleradas en medios urbanos, el caso del municipio de Franca - Sao Paulo, Brasil (Megda, 2011), la degradación ambiental y desertificación el municipio de Espinosa, Brasil (Marília \& Soares, 2011), 
el uso del suelo y la cuestión ambiental al sudoeste de Bahía, Brasil (Rodrigues et al., 2011), las divergencias y convergencias de las políticas de sustentabilidad en desarrollos hidroeléctricos en río Araguari, Minas Gerais, Brasil (Rodrigues y Da Silva, 2011), la dinámica hidrológica del río Uberabihna, Minas Gerais, Brasil (Soares et al., 2011), los procesos de sedimentación en sistemas lagunas en la Zona Norte de Costa Rica (Solano \& Salas, 2011), el factor topográfico en la Ecuación Universal de Pérdida del Suelo en la ciudad de Río Claro, Brasil (Souza \& Lupinacci, 2011), y los riesgos y vulnerabilidades ambientales del territorio de Identidade do Sisal, Bahía, Brasil (Salustiano, 2011).

Prácticamente, el $48 \%$ de los trabajos publicados referentes a riesgos naturales y antrópicos se clasifican como estudios de amenazas hidrometeorológicas o de degradación ambiental y erosión de los suelos. Dadas las condiciones tropicales de la mayoría de contextos latinoamericanos se explica que estos dos grupos de ejes temáticos tengan una representación mayor, en amenazas como las inundaciones, algunos procesos gravitacionales asociados, las sequías, los ciclones tropicales y cómo la transformación desordenada del paisaje contribuye al impacto ambiental y elevadas tasas de erosión de los suelos.

Thomas (2011) define vulnerabilidad como el nivel de exposición, fragilidad, propensión de daño y resistencia que se ofrece ante la potencial ocurrencia de amenazas y la capacidad de asimilación, ajuste y recuperación posterior dados por las condiciones socio-culturales, políticas e institucionales. Dentro de la gestión del riesgo de desastres, una variable fundamental es la vulnerabilidad, sus estudios muchas veces han sido desarrollados por profesionales de las ciencias sociales; no obstante, toman notoriedad desde la Geografía a partir de la zonificación y distribución de sus diferentes tipos a partir de 1998 hasta la actualidad.

Los trabajos de vulnerabilidad son de vital importancia en los estudios de gestión del riesgo de desastres y análisis como el de vulnerabilidad económica en áreas de riesgo por amenazas naturales en Ciudad Cortés es un ejemplo de ellos (Arroyo, 1998). Otros estudios a tomar en cuenta dentro de este eje temático son los de zonificación de la vulnerabilidad estructural y de la población ante la amenaza tecnológica del poliducto de RECOPE (Chavarría \& Camacho, 2008), la percepción geográfica de los riesgos meteorológicos que afectan en el turismo (Herbe, 2011), la 
ocupación del espacio y las problemáticas ambientales asociadas a las inundaciones en Buenos Aires, Argentina (Greco, 2011), la cartografía de vulnerabilidades en áreas urbanas de Curitiba, Brasil (Lourenco et al., 2011), la vulnerabilidad de asentamientos irregulares en Barrio Sarmiento en Buenos Aires, Argentina (Plot, 2011), los indicadores de vulnerabilidad y clasificación de áreas de riesgo ambiental en la Sierra de Periperi en Vitória de la Conquista, Bahía, Brasil (Silveira, 2011), y los índices de desarrollo distrital y su influencia con la recurrencia de inundaciones y deslizamientos en Costa Rica (Arroyo, 2014).

Con la evidente crecida en el número, frecuencia e impacto de los desastres en el mundo, se declaró la década de 1990 como el Decenio Internacional para la Reducción de los Desastres Naturales, lo que conllevó a una tendencia clara de producción de conceptos, terminologías y metodologías para el estudio de los desastres, desde el peligro/amenaza, la vulnerabilidad y el riesgo con trabajos publicados en la revista desde 1992 hasta la actualidad.

Los trabajos que tratan la epistemología de la gestión del riesgo de desastres nacen para la revista como los estudios de manejos de cuencas ante desastres naturales, caso del río Reventado (Fallas \& Gutiérrez, 1992), un mapa preliminar de amenazas geológicas de Costa Rica (Mora et al., 1992), el desastre natural como un concepto cambiante (Arroyo, 1993), las referencias conceptuales en el abordaje de los desastres naturales (Patterson, 1993). Posteriormente, se da un salto temporal hasta el estudio de Saborío (2009) con una metodología para la gestión de cuencas hidrográficas siguiendo el enfoque del riesgo integral, Barrantes y Márquez (2011) hacen un brillante análisis sobre la visión desde la geografía de la atención de desastres y la gestión del riesgo, el eje ideológico de los términos en los desastres (Calderón, 2011), riesgo y peligro en Santiago de Chile (Sánchez, 2011), una reflexión contemporánea del concepto e implicaciones de la naturaleza del desastre (Villarreal, 2011), la gestión integral del riesgo en la planificación urbana de San Luis Potosí en México (Palacio, 2011), y la participación popular en el enfrentamiento de desastres ambientales, caso de Santa Catarina en Brasil (Ferreira et al., 2011).

Cabe resaltar que la mayoría de trabajos publicados son de Costa Rica, dada la naturaleza y orígenes de la revista misma con 56 publicaciones (49\% del total); posteriormente, se ubican Brasil con 28 trabajos, 
9 trabajos conceptuales sin una localización precisa, y seguido México, Chile, Argentina y otros. Esto demuestra la fuerza de ciertas escuelas geográficas latinoamericanas como lo son Brasil, México, Chile y Argentina que tratan de permear otras partes de la región.

Los autores con más trabajos en la temática de riesgos naturales y antrópicos en la Revista Geográfica de América Central han sido Luis Nelson Arroyo (9 trabajos), Wilhem Vahrson (7), Gustavo Barrantes y Ora Patterson (5), F.D. Bennett (4), Iliana Arauz, Jorge Brenes, Nury Cartín, Jorge Fallas, Gonzalo Hernández, Carlos Montero y L. Morales (3). Es importante indicar que la mayoría de estos autores son Geógrafos además, esto permite analizar el aporte de la Geografía en las investigaciones realizadas con la Gestión de Riesgos en diferentes momentos.

\section{Conclusiones}

La clasificación de las temáticas de los artículos relacionados a riesgos naturales y antrópicos en la Revista Geográfica de América Central indica los tipos de estudios que predominan en Costa Rica; además, marcan la influencia que tienen escuelas del conocimiento latinoamericano como lo son Brasil, México y Chile en la manera de abordar los estudios en gestión del riesgo de desastres. Las condiciones de exposición a procesos naturales extraordinarios como lo son la sismicidad, el vulcanismo, los fenómenos atmosféricos y la degradación ambiental aunada a la vulnerabilidad de la sociedad latinoamericana hacen estos temas de vital importancia en el desarrollo de nuestros pueblos.

A través de la revista se logra apreciar un crecimiento de los trabajos en temáticas de riesgos, con un marcado aumento de los estudios en riesgos antrópicos a partir de la década de 1990. Es por ello que se insta a que se integre cada vez más los estudios con un enfoque más holístico, propio de la Geografía y tendencias de entender el espacio como un ente vivo y dinámico donde convergen tanto los fenómenos físico-naturales como los sociales.

A partir de esta investigación se propone que los estudios de la amenaza sísmica involucren su asociación con el ordenamiento territorial más allá de la mera explicación de los procesos internos de la Tierra, sino sus implicaciones en el desarrollo nacional, regional y local. Por otro lado, se insta al desarrollo de trabajos sobre volcanes con probable reactivación en Costa Rica y la región latinoamericana, ya que muchas veces se centra 
la atención únicamente a los volcanes en procesos eruptivos dejando de lado los latentes. Desde el estudio de los movimientos en masa se debe avanzar a metodologías de zonificación de la susceptibilidad y probabilidad de procesos de ladera en Costa Rica, más allá de metodologías como la Mora-Vahrson que aún no ha sido superada dada la versatilidad de su aplicación en la región.

Se debe hacer una clara diferenciación conceptual entre trabajos asociados con variabilidad climática, calentamiento global y cambio climático, porque muchas veces estos estudios se tratan de simples fenómenos hidrometeorológicos donde no intervienen dinámicas multidecadales, la acción del ser humano, ni las escalas milenarias de cambios asociados con las variaciones astronómicas de la Tierra. Deben ampliarse los estudios de degradación ambiental desde la erosión, contaminación y alteración de los suelos, los cuerpos de agua y las condiciones atmosféricas en entornos no sólo urbanos sino rurales y su asociación directa con el cambio en el uso del suelo y la falta de ordenamiento territorial efectivo.

Los trabajos de vulnerabilidad son de gran necesidad en el contexto latinoamericano, en la actualidad se ha desarrollado una gran cantidad de cartografías de amenazas naturales, que a pesar de necesitar mayor detalle, hay en abundancia. No obstante, los trabajos integrados de vulnerabilidad y riesgos aún son escasos en la región, lo que no permite muchas veces terminar de integrar los esfuerzos desde las ciencias físicas y sociales.

\section{Agradecimientos}

A los estudiantes Erich Mata, Sofía Bolaños y Gabriel Alvarado por haber colaborado con la recopilación, ordenación y tratamiento de la información de este trabajo.

\section{Referencias}

Alcántara-Ayala, I. (2000). Landslides: deslizamientos o movimiento del terreno? Definición, clasificaciones y terminología. Investigaciones Geográficas: Boletín del Instituto de Geografía-UNAM 41:7-25. Alcántara-Ayala, I. (2002). Geomorphology, natural hazards and prevention of natural disasters in developing countries. Geomorphology, 47, 107-124. 
Alfaro, M. (1992). Caracterización de la precipitación húmeda en el Parque Nacional Volcán Poás, Costa Rica. Revista Geográfica de América Central, 25-26, 73-84.

Arroyo, N. (1992a). Influencia antrópica y deslizamientos en Ciudad Colón y Puriscal. Revista Geográfica de América Central, 25-26, 265-279. Arroyo, N. (1992b). Riesgo por inundaciones en Costa Rica. Revista Geográfica de América Central, 25-26, 373-384.

Arroyo, L. (1993). Desastre natural: un concepto cambiante. Revista Geográfica de América Central , 28, 11-14.

Arroyo. N. (1996). Sensores remotos fotográficos: zonificación por inundaciones en las cuencas bajas de los ríos Parrita y Limoncito: una caracterización socioespacial mediante fotos aéreas y mapas. Revista Geográfica de América Central, 32-33, 165-181.

Arroyo, N. (1998). Vulnerabilidad económica en Áreas de Riesgo por Amenazas Naturales: una aproximación metodológica para su estimación, el caso de Ciudad Cortés, Costa Rica. Revista Geográfica de América Central, 36, 121-133.

Arroyo, L. N. (2008). Caracterización geomorfológica, amenazas naturales y restricciones de uso de la tierra en Esparza, Costa Rica: Un enfoque de ordenamiento territorial para el plan regulador del cantón. Revista Geográfica de América Central, 41, 1-28.

Arroyo, L. N. (2011). Costa Rica: Análisis de la incidencia espacial de inundaciones y deslizamientos por provincias y cantones, años 2000-2006. Revista Geográfica de América Central, 47, 97-126.

Arroyo, L. N., Salgado, D. \& Moraga, C.V. (2011). Costa Rica: análisis de la incidencia relacionada con inundaciones y deslizamientos por provincias y cantones, década 1999 al 2009. Revista Geográfica de América Central, (Número Especial EGAL 2), 1-16.

Arroyo, L. N. (2014). Gestión del riesgo y los índices de desarrollo distrital: un enfoque de casos. Revista Geográfica de América Central, $52,81-111$.

Alvarado, G., Matumoto, T., Borgia, A. \& Barquero, R. (1992). Síntesis geovulcanológica del Arenal (Costa Rica): 20 años de continua actividad eruptiva (1968-1988). Revista Geográfica de América Central, 25-26, 413-459. 
Bankoff, G. (2004). Time is of the essence: disasters, vulnerability and history. International Journal of Mass Emergencies and Disasters November 2004, 22(3), 23-42

Barquero, J., Sáenz, R. \& Fernández E. (1992). El Volcán Poás, Históricamente un volcán activo. Revista Geográfica de América Central, 25-26, 241-246.

Barrantes, G., Segura, A. \& Walcott, K. (2008). Percepción y prevención del riesgo por lahar en los diques de Cartago. Revista Geográfica de América Central, 42, 83-96.

Barrantes, G., Barrantes, O. \& Núñez, O. (2011). Efectividad de la metodología Mora-Vahrson modificada en el caso de los deslizamientos provocados por el terremoto de Cinchona, Costa Rica. Revista Geográfica de América Central, 47, 141-162.

Barrantes, G. \& Vargas J. (2011). La zonificación de amenaza por inundación como herramienta para el ordenamiento territorial en el valle del Río Sixaola. Revista Geográfica de América Central, 46, 67-85.

Barrantes, G. \& Márquez, R. (2011). De la atención del desastre a la gestión del riesgo; una visión desde la geografía. Revista Geográfica de América Central, 47, 15-38.

Barrantes, G., Jiménez, C. \& Ocón, A. J. (2013). Deslizamientos provocados por el terremoto de Cinchona de 2009, Costa Rica. Revista Geográfica de América Central, 51, 89-100.

Bennett, F. \& Raccichini, S. (1977a). Las erupciones del volcán Arenal, Costa Rica. Revista Geográfica de América Central, 5-6, 7-35.

Bennett, F. \& Raccichini, S. (1977b). Nuevos Aspectos de las Erupciones del Volcán Poas. Revista Geográfica de América Central, 5-6, 37-53. Bennett, F., \& Wright, H. (1979). Nuevos niveles de azufre en el cráter del Volcán Poas. Revista Geográfica de América Central, 9-10, 79-84.

Bennett, F. (1980). Fumarolas y pozos subacuáticos de azufre en el Volcán Poas. Costa Rica. Revista Geográfica de América Central, 11-12, 125-129.

Brenes, J. (1992). Estudio preliminar de la sismicidad de la costa Caribe de Costa Rica, con énfasis en la fuente sísmica Parismina. Revista Geográfica de América Central, 25-26, 247-264. 
Borgia, A. \& Cigolini, C. (1980). Consideraciones sobre la viscosidad de las lavas y la estructura de las coladas del Volcán Arenal. Costa Rica. Revista Geográfica de América Central, 11-12, 131-140.

Calderón, G. (2011). Lo ideológico de los términos en los desastres. Revista Geográfica de América Central, (Número Especial EGAL 2), $1-16$.

Campos, R., Gomes, E., Rebello da Cunha, A. \& Nonato, R. (2011). Análise geográfica sobre erosão de margens e movimentos de massa na comunidade do Divino E. Santo - AM (Brasil). Revista Geográfica de América Central, (Número Especial EGAL 2), 1-17.

Cardoso, D. M. (2011). O reordenamento da Calha do Rio Jequitinhonha no controle de inundação na cidade de Belmonte na Bahia, Brasil. Revista Geográfica de América Central, (Número Especial EGAL 2), 1-13.

Cartín, N. \& Ellenberg, L. (1986). Los dos lados de la Cordillera Central de Costa Rica. Revista Geográfica de América Central, 19-20, 235-245.

Cassertano, L. (1979). Cálculo de las magnitudes máxima y mínima de los sismos. Revista Geográfica de América Central, 9-10, 85-118.

Cassol, M. L., \& Floriani, N. (2011). Processos erosivos no subsistema "Terras-de-plantar" de um faxinal paranaense sob cultivo de fumo integrado. Revista Geográfica de América Central, (Número Especial EGAL 2), 1-19.

Cevo, H. (1974). Manifestaciones volcánicas en Aysén (Chile). Revista Geográfica de América Central, 1, 51-75.

Chavarría, N. M. \& Camacho, C.M. (2008). Propuesta metodológica para la zonificación de la vulnerabilidad estructural y de la población ante la presencia de una amenaza tecnológica: El caso del poliducto de RECOPE, Costa Rica. Revista Geográfica de América Central, 42, 57-82.

Chacón, R. (1993). Aspectos de la influencia del fenómeno del niño en el clima costarricense. Revista Geográfica de América Central, 27, 53-66.

Correia, L. E., De Souza, M., De Arruda, T. \& Facciulo D. (2011). Investigação do histórico das obras de contenção em feição erosiva, Município de Terra Rica, Paraná, Brasil. Revista Geográfica de América Central, Número Especial EGA 2), 1-15. 
Cortese, S., Passos, E., Muratori, A. M. \& Cacia, E. (2011). Identificação de riscos ambientais na bacia do Rio Verde - RMC - Paraná, Brasil, por meio de um sistema de informação geográfica. Revista Geográfica de América Central, (Número Especial EGAL 2), 1-16.

Dos Santos, A., Alves, W., Araújo, H. \& Nascimento, J. (2011). Riscos geomorfológicos no Bairro Santa Maria Aracaju/se. Revista Geográfica de América Central, (Número Especial EGA 2), 1-8.

Duarte, E. \& Fernández, E. (2011). Callejones de acidificación en tres volcanes activos de Costa Rica. Revista Geográfica de América Central, 47, 127-130.

Duarte, E. (2014). Las cuatro erupciones freáticas recientes del Volcán Turrialba (2010-2013) Una por año. Revista Geográfica de América Central, 52, 139-161.

Escuela de Ciencias Geográficas de la Universidad Nacional. (1977). La Actividad del Volcán Irazú en 1723. Revista Geográfica de América Central, 5-6, 203-210.

Espinosa, M. A. (2011). Cambio Global: Cambio climático global, globalización y nueva división internacional del trabajo. Revista Geográfica de América Central, (Número Especial EGAL 2), 1-20.

Fallas, J. (1992a). CFA88: un análisis versátil para el análisis de eventos hidrometeorológicos extremos. I. Teoría. Revista Geográfica de América Central, 25-26, 99-113.

Fallas, J. (1992b). CFA88: un análisis versátil para el análisis de eventos hidrometeorológicos extremos. II. Aplicación. Revista Geográfica de América Central, 25-26, 85-97.

Fallas, J. \& Gutiérrez, M. (1992). Manejo de cuencas ante desastres naturales: cuenca alta del río Reventado, Cartago. Revista Geográfica de América Central, 25-26, 341-353.

Fassone, R. (1992). Desastre, una verdadera calamidad... se están agotando nuestros suelos y nuestras aguas. Revista Geográfica de América Central, 25-26, 139-151.

Ferreira, D., Albino, L. \& Cardoso, M. J. (2011). Participação popular na prevenção e enfrentamento de desastres ambientais: resultado de um estudo piloto em Santa Catarina, Brasil. Revista Geográfica de América Central, (Número Especial EGAL 2), 1-17. 
Freitas, E. \& Da Anunciação, V. (2011). A vulnerabilidade espacial climática na cidade de Aquidauana - MS/Brasil. Revista Geográfica de América Central, (Número Especial EGAL 2), 1-19.

Galvão, N. \& Ribeiro, J. (2011). O processo de desertificação no sítio Manduri-Pé Brasil. Revista Geográfica de América Central, (Número Especial EGAL 2), 1-13.

González, H., González, C. \& Rosales, L. (1986). Plan para incorporar el riesgo sísmico a los planes de uso del terreno. Revista Geográfica de América Central, 19-20, 185-201.

Govaere, J. (1974). Mapa de Isoyetas de la Tormenta del 28-29 de Agosto de 1973, Cuenca del Rio Grande de Terraba. Revista Geográfica de América Central, 1, 21-28.

Granados, R., Aguilar, G., Díaz, G. \& Medina, M. (2011). Alteraciones de los indicadores agroclimáticos en años con presencia del fenómeno El Niño en la región Centro-Occidente de México. Revista Geográfica de América Central, (Número Especial EGAL 2), 1-16.

Greco, M. G. (2011). Ocupación del espacio y problemáticas ambientales: las inundaciones en la ciudad de Buenos Aires. Revista Geográfica de América Central, (Número Especial EGAL 2), 1-15.

Güendel, F., Montero, C., González, V., Segura, J. \& Brenes, J. (1992). Actividad sísmica de cráter superficial registrada por la red sismológica del OVSICORI-UNA en la hoja de San José 1:200 000 entre abril 1984-julio 1988. Revista Geográfica de América Central, 2526, 11-44.

Herbe, X. (2011). Percepção geográfica dos riscos meteorológicas que afetam o turismo. Revista Geográfica de América Central, (Número Especial EGAL 2), 1-9.

Hernández, G. (1995). Modelo Geomorfométrico aplicado al estudio de la erosión en cárcavas. Revista Geográfica de América Central, 31, 59-95.

Hernández, G., Ruiz, A., Barrantes, G. \& Díaz, J. (1995). Erosión de suelos en Cerbatana de Puriscal 1990-1995. Revista Geográfica de América Central, 31, 97-123.

Hernández, G. \& Ruiz, A. (1998). Modelaje de la Erosión de Suelos en Costa Rica mediante el Modelo WEPP. Revista Geográfica de América Central, 36, 11-36. 
Hernández, M., Carrasco, G. \& Alfaro, G. (2011). Degradación del suelo en una zona semiárida tropical de México. Revista Geográfica de América Central, (Número Especial EGAL 2), 1-14.

Hernando, L. \& Arauz, I. (1994). Deslizamientos en la cuenca del río Banano, una consecuencia del terremoto de abril de 1991. Revista Geográfica de América Central, 30, 47-63.

Lavell, A. (2005). Los conceptos, estudios y práctica en torno al tema de los riesgos y desastres en América Latina: evolución y cambio, 1980-2004: el rol de LA RED, sus miembros y sus instituciones de apoyo. FLACSO, Facultad Latinoamericana de Ciencias Sociales, Secretaría General. (pp. 66)

Lourenco, N., Briski, S. \& Prates, V. (2011). O mapeamento das vulnerabilidades ambientais em áreas urbanas da região metropolitana de Curitiba/PR como proposta para minimização de desastres. Revista Geográfica de América Central, (Número Especial EGAL 2), 1-14.

Loyola, E., Medellín, P., Avalos, J. \& Aguilar, M. (2011). Cambio climático y variabilidad en la dinámica de los ecosistemas de Wirikuta, Municipio de Catorce (1950-2010). Revista Geográfica de América Central, (Número Especial EGAL 2), 1-18.

Madrigal, D., Franco, R., Miguel, L., Arcelia, M. \& Reyes, A. (2010). Caracterización de las regiones tectónicas del estado de México a través de la aplicación de geotecnologías. Revista Geográfica de América Central, (Número Especial 1), 15-36.

Marília, A. \& Soares, M. I. (2011). Degradação ambiental e desertificação no semiárido mineiro: um estudo sobre o Município de Espinosa (MG). Revista Geográfica de América Central, (Número Especial EGAL 2), 1-16.

Martínez, C. (2011). Análisis comparativo de cambios históricos de la línea litoral en bahías chilenas: orientaciones al manejo costero. Revista Geográfica de América Central, (Número Especial EGAL 2), 1-17.

Martínez, C., Rojas, O., Jaque, E., Quezada, J., Vázquez, D. \& Belmonte, A. (2011). Efectos territoriales del tsunami del 27 de febrero de 2010 en la costa de la región del Bio-Bío, Chile. Revista Geográfica de América Central, (Número Especial EGAL 2), 1-16.

Maskrey, A. (1993). Los desastres no son naturales. (LA RED) Red de estudios sociales en prevención de desastres en América Latina. Tercer Mundo Editores. Bogotá, Colombia. (pp. 137) 
Megda, O. (2011). Impactos das erosões aceleradas em meio urbano: o caso do Município de Franca - São Paulo. Revista Geográfica de América Central, (Número Especial EGAL 2), 1-19.

Meneses, B. M. (2013). O impacto dos incêndios florestais na Perda de solo por erosão hídrica na Serra de Santa Helena. Revista Geográfica de América Central, 51, 215-232.

Merino, J. (1975). El Terremoto que destruyó la ciudad de Guatemala. Revista Geográfica de América Central, 3, 65-70.

Montero, C., Rodríguez, H. \& Moraga, J. (1997). Aplicación del Modelo de Simulación de Intensidades Sísmicas Privado en la Microzonificación de Costa Rica. Revista Geográfica de América Central, 34, 121-141.

Mora, R. \& Morales, L. (1992). La susceptibilidad a la inestabilidad de laderas en la zona norte de Alajuela y el terremoto de Fraijanes de 1888. Efectos y previsión. Revista Geográfica de América Central, 25-26, 311-322.

Mora, S. (1977). Estudio geológico del Cerro Chopo. Revista Geográfica de América Central, 5-6, 189-199.

Mora, S., Alvarado, G. \& Morales, L. (1992). Mapa preliminar sintético de amenazas geológicas de Costa Rica. Su importancia en el desarrollo de la infraestructura civil. Revista Geográfica de América Central, 25-26, 355-371.

Moraga, J. C. (2010). Evaluación del riesgo ante incendios forestales en la cuenca del Río Tempisque, Costa Rica. Revista Geográfica de América Central, 45, 33-64.

Morales, L., Montero, W. \& Madrigal, R. (1992). El terremoto de Fraijanes de 1888 y el sistema de fallas de Alajuela, implicaciones del peligro sísmico potencial para el Valle Central Occidental. Revista Geográfica de América Central, 25-26, 227-240.

Ortiz, M. \& Vidal, R. (2011). Población afectada por fenómenos climáticos extremos. Revista Geográfica de América Central, (Número Especial EGAL 2), 1-15.

Oliveira, P. \& Baptista, S. (2011). Juiz de Fora: inundações, saneamento e ordenamento territorial. Revista Geográfica de América Central, (Número Especial EGAL 2), 1-15. 
Palacio, G. (2011). La gestión integral del riesgo como estrategia para la planificación urbana en San Luis Potosí, México. Revista Geográfica de América Central, (Número Especial EGAL 2), 1-9.

Patterson, O. (1992). Riesgo por sequías en Costa Rica. Revista Geográfica de América Central, 25-26, 385-411.

Patterson, O. (1993). Referencias conceptuales en el abordaje de los desastres naturales. Revista Geográfica de América Central, 28, 15-23.

Pereira, L., Guedes, A., Linhares, C. \& Zanella, M. E. (2011). Estudo da gênese dos eventos pluviométricos, iguais ou superiores a $100 \mathrm{~mm} / 24 \mathrm{~h}$, na cidade de Fortaleza - CE, no período de 1996 - 2008. Revista Geográfica de América Central, (Número Especial EGAL 2), 1-20.

Pereira, A. L. \& Oliveira, R. (2011). Impactos da urbanização no sistema hidrológico - inundações no Município de Guarujá - SP. Revista Geográfica de América Central, (Número Especial EGAL 2), 1-12.

Plot, B. (2011). Vulnerabilidad en los asentamientos irregulares del AMBA: análisis de caso, Barrio Sarmiento. Revista Geográfica de América Central, (Número Especial EGAL 2), 1-17.

Protti, J., Güendel, F., Montero, C. \& Brenes, J. (1992). Interpretación preliminar de la secuencia sísmica de Potrero Grande (Marzo de 1988) y su relación con la secuencia sísmica de Sierpe (marzo de 1987). Revista Geográfica de América Central, 25-26, 281-289.

Quesada, M. E. (2001). Comportamiento hidrológico estacional y su relación con el ENOS en la parte alta de la cuenca del río Tárcoles, Costa Rica. Revista Geográfica de América Central, 39(1-2), 93-111.

Ramírez, P. (1992). Descripción de situaciones climatológicas que pueden producir desastres en Costa Rica. Revista Geográfica de América Central, 25-26, 153-169.

Rodríguez, A. (1992). Efecto de la variación del coeficiente de escorrentía en la frecuencia de las avenidas. Revista Geográfica de América Central, 25-26, 209-225.

Rodrigues, M., Oliveira, E. \& Maia, E. (2011). O uso do solo e a questão ambiental na região sudoeste da Bahia - Brasil. Revista Geográfica de América Central, (Número Especial EGAL 2), 1-15.

Rodrigues, H. \& Da Silva, V. (2011). Divergências e convergências nas políticas de sustentabilidade de empreendimentos hidrelétricos: efeitos 
socioespaciais no Rio Araguari-Minas- Gerais - Brasil. Revista Geográfica de América Central, (Número Especial EGAL 2), 22.

Saborío, J. (2009). Metodología para la gestión de cuencas hidrográficas siguiendo el enfoque del riesgo integral. Revista Geográfica de América Central, 42, 25-35.

Sáenz, R. (1977). Erupción del Volcán Arenal en el año 1968. Revista Geográfica de América Central, 5-6, 149-188.

Salustiano, A. (2011). O processo de desmatamento do bioma Caatinga: riscos e vulnerabilidades socioambientais no território de Identidade do Sisal, Bahia. Revista Geográfica de América Central, (Número Especial EGAL 2), 1-13.

Sandoval, L. F. \& Chacón, W. (2011). Riesgo de inundación en el distrito de Cahuita, Talamanca y la percepción de la población a estos eventos para la determinación de algunas medidas preventivas, año 2010. Revista Geográfica de América Central, 47, 255-224.

Sánchez, R. (2011). Riesgo y peligro en las ciudades Latinoamericanas, el caso del área metropolitana de Santiago, Chile. Revista Geográfica de América Central, (Número Especial EGAL 2), 1-18.

Segura, J., Quintero, R., Burgoa, B., \& Jiménez, W. (2014). Análisis de la actividad sísmica en Costa Rica durante el 2010 y resumen de los eventos sísmicos más importantes presentados en Costa Rica de 1983 - 2012. Revista Geográfica de América Central, 52, 70-102.

Segura, L. A., Saracho, M. A. \& Nieva, N. Y. (2011). Riesgo de contaminación de los recursos hídricos por actividades antropogénicas en el departamento capital, provincia de Catamarca, República Argentina. Revista Geográfica de América Central, (Número Especial EGAL 2), 1-15.

Silva, T., Carvalho, J. \& Vieira, U. D. (2011). Geografía física, riesgos socioambientales y cambio climático. Revista Geográfica de América Central, (Número Especial EGAL 2), 1-13.

Silveira, P. (2011). Indicadores de vulnerabilidade e classificação de áreas de risco ambiental na vertente sul da Serra do Periperi em Vitória da Conquista - Bahia - Brasil. Revista Geográfica de América Central, (Número Especial EGAL 2), 1-15.

Soares, A. M., Dos Santos, R. \& Almeida, R. (2011). A dinâmica hidrológica na bacia do alto curso do Rio Uberabinha em Uberaba, Minas 
Gerais, Brasil. Revista Geográfica de América Central, (Número Especial EGAL 2), 1-17.

Solano, F., \& Salas, D. (2011). Procesos hidrodinámicos de sedimentación en sistemas lagunares continentales en la Zona Norte de Costa Rica. Revista Geográfica de América Central, (Número Especial EGAL 2), 1-22.

Somarribas, L. \& Patterson, O. (1997a). Degradación de recursos, pobreza y salud en el cantón de Puriscal (Parte I). Revista Geográfica de América Central, 34, 57-85.

Somarribas, L. \& Patterson, O. (1997b). Degradación de recursos, pobreza y salud en el cantón de Puriscal (Parte II). Revista Geográfica de América Central, 34, 87-102.

Soto, G. \& Paniagua, S. (1992). La Cordillera Volcánica Central (Costa Rica): sus peligros potenciales y prevenciones. Revista Geográfica de América Central, 25-26, 291-304.

Souza, L. \& Lupinacci, C. M. (2011). A importância da geração do fator topográfico (LS) da EUPS para modelagem erosiva de bacia hidrográfica. Revista Geográfica de América Central, (Número Especial EGAL 2), 1-13.

Toscana, A. (2006). Los paisajes del desastre. Doctorado en Geografía. Posgrado en Geografía. Universidad Nacional Autónoma de México. (pp.239)

Tanizaki, K. \& Azevedo, F. (2011). Eventos sísmicos no Brasil, percepção de risco de discentes de geografia frente a eventos pseudo - remotos. Revista Geográfica de América Central, (Número Especial EGAL 2), 1-17.

Thomas, J. (2011). Gestión social del riesgo ante amenazas naturales en ciudades portuarias. Estudios de caso: Buenaventura (Colombia) y Manzanillo (México). Tesis de Doctorado en Geografía. Facultad de Filosofía y Letras, UNAM. México D.F., México. 366 pp.

Uboldi, J., Laucirica, J., Michalijos, P. \& Mancino, M. (2011). Cambio climático, turismo y riesgo de incendios forestales, de pasturas y de su interfase, en el sudoeste Bonaerense, su análisis con geotecnologías. Revista Geográfica de América Central, (Número Especial EGAL 2), 1-15.

Vahrson, W. (1992). Distribuciones de los periodos de retorno de tres eventos de lluvias extremas en Costa Rica. Revista Geográfica de América Central, 25-26, 193-207. 
Vahrson, W. \& Cartín, N. (1992). Deslizamientos en la cuenca media y superior del río Reventado, Cartago, Costa Rica. Revista Geográfica de América Central, 25-26, 171-192.

Vahrson, W. Cartín, N. \& Patterson, O. (1992a). Las avalanchas e inundaciones de Julio de 1987 en las cuencas del río Grande de Orosi y el río Pejibaye: un estudio de caso. Revista Geográfica de América Central, 25-26, 323-340.

Vahrson, W., Romero, M., Arauz, I., Sánchez, S. \& Chacón, R. (1992b). Análisis probabilístico de lluvias intensas en Costa Rica. Revista Geográfica de América Central, 25-26, 45-58.

Vahrson, W., Saborío, F. \& Hernando, L. (1992c). Avalanchas en la Sierra Volcánica Central. El caso del río Ciruelas. Revista Geográfica de América Central, 25-26, 59-72.

Vahrson, W. (1993). Erosión y conservación de suelos en Costa Rica. Algunos resultados del taller de erosión de suelos. Revista Geográfica de América Central, 27, 187-196.

Vahrson, W. \& Arauz, I. (1993). Aspectos morfoclimatológicos de la región bahía de Culebra, bahía El Coco, Guanacaste, Costa Rica. Revista Geográfica de América Central, 27, 67-87.

Villareal, I. (2011). La naturaleza del desastre: una reflexión contemporánea del concepto y sus implicaciones. Revista Geográfica de América Central, (Número Especial EGAL 2), 1-13.

Volker, E. \& Casagrande, M. P. (2011). Caracterização dos movimentos de massa na microbacia do rio Itoupava, Município de Rio do Sul / SC - Brasil. Revista Geográfica de América Central, (Número Especial EGAL 2), 1-17.

White, G. (1942). Human Adjustment to Floods: A Geographical Approach to the Flood Problem in the United States. Chicago: University of Chicago, Department of Geography.

White, G. (1974). Natural Hazards. New York: Oxford University Press.

Zárate, E. (1992). Clasificación de fenómenos meteorológicos causantes de desastres naturales según escalas temporales y espaciales. Revista Geográfica de América Central, 25-26, 115-131.

Zocche, N. \& Peralta, D. (2011). Análise geomorfológica dos movimentos de massa ocorridos na MT 170 Juina/Castanheira. Revista Geográfica de América Central, (Número Especial EGAL 2), 1-17. 